\title{
Two-dimensional correlation spectroscopy of two-exciton resonances in semiconductor quantum wells
}

\author{
Lijun Yang and Shaul Mukamel \\ Chemistry Department, University of California, \\ Irvine, CA 9269\%-2025, United States
}

(Dated: November 5, 2018)

\begin{abstract}
We propose a three-pulse coherent ultrafast optical technique that is particularly sensitive to two-exciton correlations. Two Liouville-space pathways for the density matrix contribute to this signal which reveals double quantum coherences when displayed as a two-dimensional correlation plot. Two-exciton couplings spread the cross peaks along both axes, creating a characteristic highly resolved pattern. This level of detail is not available from conventional one-dimensional four-wave mixing or other two-dimensional correlation spectroscopy signals such as the photo echo, in which two-exciton couplings show up along a single axis and are highly congested.

PACS numbers: 78.47.+p,78.67.De, 42.50.Md,71.35.Cc
\end{abstract}


Investigating the correlations of multiple excitons in semiconductors is a challenging many-body problem that had drawn considerable theoretical $[1,2,3,4,5]$ and experimental $[6,9]$ attention. Correlations of two-excitons beyond Hartree-Fock (HF) may result in either a red-shift (two-exciton binding energy,TBE) or a blue-shift (two-exciton scattering energy, TSE). In GaAs semiconductor quantum wells both couplings are a few meVs and may not be easily resolved. Two-exciton formation has been reviewed in Refs.[6, 7, 8]. Coherent ultrafast four-wave mixing (FWM) [6] provides a direct probe for two-exciton correlations in quantum wells. The best evidence for bound two-excitons and the most accurate TBE in GaAs quantum wells are obtained by time integrated FWM (TIFWM), where signals are displayed as a function of a single (time or frequency) variable. $[6,10,11]$ Quantum beats were observed in the negative-delay two-pulse signal along $2 \mathbf{k}_{b}-\mathbf{k}_{a}$ where $\mathbf{k}_{b}$ arrives first, and in the positive-delay three-pulse signal along $\mathbf{k}_{a}+\mathbf{k}_{b}-\mathbf{k}_{c}\left(\mathbf{k}_{c}\right.$ comes after $\left.\mathbf{k}_{b}\right)$. Many attempts have been made to enhance the spectral resolution by displaying FWM signals versus two time variables.[12, 13, 14]

Multidimensional analysis of coherent signals is commonly used in NMR to study correlations between spins.[15] These techniques were recently extended to the femtosecond regime[16] and applied to several chemical and biological systems.[17, 18] Three ultrashort laser pulses generate a signal which is heterodyne-detected by a fourth pulse in a chosen phase-matching direction (Fig. 1 top left). Three time delays $\left(t_{1}, t_{2}, t_{3}\right)$ can be controlled between the chronologically ordered pulses, $\mathbf{k}_{1}, \mathbf{k}_{2}, \mathbf{k}_{3}$ and the heterodyne pulse $\mathbf{k}_{s}$. For an excitonic system the signal can be generated along the phase-matching directions, $-\mathbf{k}_{1}+\mathbf{k}_{2}+\mathbf{k}_{3}, \mathbf{k}_{1}-\mathbf{k}_{2}+\mathbf{k}_{3}$ and $\mathbf{k}_{1}+\mathbf{k}_{2}-\mathbf{k}_{3}$. We shall denote these three techniques as $S_{\mathbf{I}}, S_{\text {II }}$ and $S_{\text {III }}[19,20]$ respectively. The time-domain nonlinear response is given by combinations of multi-time correlation functions which depend on the time delays $t_{1}, t_{2}$ and $t_{3}$. Displaying the signal as a function of two time delays (or their conjugate frequencies) while holding the third fixed gives the two-dimensional correlation spectroscopy (2DCS) signals. Note that $t_{1}, t_{2}$ and $t_{3}$ are positive. This is different from conventional FWM where there is no fixed time ordering among the $\mathbf{k}_{a}, \mathbf{k}_{b}, \mathbf{k}_{c}$ pulses and their delays can be either positive or negative. We shall show that controlling the time ordering in 2DCS is critical for the unambiguous identification of many-body correlations. $S_{\mathbf{I}}$ (photon echo) signals were recently reported in GaAs heterostructures[21, 22, 23, 24]. In this work, we demonstrate that the $S_{\text {III }}$ technique, when displayed in a proper projection plane, can access electronic 
correlations beyond HF such as bound and unbound two-excitons with very high resolution, not available in 1D FWM and any other 2DCS techniques.

We employ a multi-band 1D tight-binding Hamiltonian[25, 26] for calculating the $S_{\text {III }}$ signal. It includes a simplified Coulomb interactions and accounts for the heavy-hole (HH), light-hole (LH) excitons and their continuum states in a tractable way. This model has been successfully used to reproduce many $1 \mathrm{D} \mathrm{FWM}[26]$ and the recent $S_{\mathbf{I}} 2 \mathrm{DCS}[23]$ experiments. For clarity we focus on the $\mathrm{HH}$ spectral region. With near-resonant excitations, only two terms contribute to the third-order optical response in the phase-matching direction $\mathbf{k}_{1}+\mathbf{k}_{2}-\mathbf{k}_{3}$. These are represented by the double-sided Feynman diagrams shown in the bottom of Fig. 1(left), where $e$ and $f$ represent respectively $\mathrm{HH}$ exciton and two-exciton manifolds and $g$ represents ground state (top right of Fig. 1) The rules of these diagrams are given in Ref. [19].

During $t_{1}$ and $t_{2}$, both pathways are respectively in a single $\left(\rho_{e g}\right)$ and double $\left(\rho_{f g}\right)$ quantum coherences. They differ only in the third interval $t_{3}\left(\rho_{e g}\right.$ for (i) and $\rho_{f e}$ for (ii)). Signatures of two-exciton couplings thus show up along $t_{3}$ and $t_{2}$ but not $t_{1}$. To enhance the resolution, we therefore look at $\left(t_{3}, t_{2}\right)$ correlation plots. We shall display the signal by a double Fourier transform with respect to $t_{3}$ and $t_{2}$ with the corresponding conjugate frequencies $\Omega_{3}$ and $\Omega_{2}$, holding $t_{1}$ fixed. The expected $2 \mathrm{DCS}, \mathrm{S}_{\mathbf{I I I}}\left(\Omega_{3}, \Omega_{2}, t_{1}\right)$, is schematically sketched at the bottom of Fig. 1. The individual contributions of pathways (i) and (ii) are denoted by the symbols given at the bottom of each Feynman diagram. $f$ can be either red-shifted (bound) $f=2 e-\Delta_{b}$, un-shifted $f=2 e$ or blue-shifted (scattering) $f=2 e+\Delta_{s}$ relative to twice the HH exciton energy, 2e. Red(blue) energy shift corresponds to two correlated excitons with opposite(same) spins. Open circles represent un-shifted two-excitons and solid (open) symbols represent red(blue) shifted two-excitons. Energy shifts from dressed states are negligible in the present weak-field $\chi^{(3)}$ limit and thus the HF (mean-field) contribution gives energy-unshifted two-excitons.

We now examine the five peaks in Fig. 1. We note that the peaks resulting from (i), are only spread along $\Omega_{2}$ and form a vertical pattern since it has eg resonances along $\Omega_{3}$, while for (ii) the peaks are spread along both axes (diagonal pattern). Four properties make this signal particularly useful for resolving various two-excitons and even the detailed structure of the two-exciton continuum. First, along $t_{2}$ there are only $f g$ resonances and thus the $\Omega_{2}$ axis provides a clean projection for two-excitons, without interference from 
eg resonances. Second, the two-exciton correlation energy can be obtained from a single peak. $\Delta_{b}$ is extracted from the solid square at $\left(e, 2 e-\Delta_{b}\right)$ and $\Delta_{s}$ from the open square at $\left(e, 2 e+\Delta_{s}\right)$. In TIFWM, in contrast, $\Delta_{b}$ is determined by subtracting the energies of two peaks. However, the HF peak (the circle) is not always clearly visible for certain pulse polarizations and laser detunings. Third, the squares from (i) for different $f$ are generally much stronger than triangles from (ii). Thus it is sufficient to get the two-exciton energies from the well-resolved and strong (i) peaks (squares) along $\Omega_{2}$ even when the triangles are not resolved. Fourth, both axes $\Omega_{2}$ and $\Omega_{3}$ involve two-exciton resonances. Along $\Omega_{2}$, there are only $f g$ resonances, while along $\Omega_{3}$ there is both $e g$ (i) and $f e$ (ii). Spreading two-exciton resonances along both axes is critical for the high resolution of two-excitons. Along $\Omega_{3}$ the two-exciton contribution $(f e$ in (ii) ) is weaker than the single-exciton one $(e g$ in (i)) and thus may not be easily resolved. However, we shall show later that even this weak signature along $\Omega_{3}$ is crucial for achieving high resolution when combined with the information from the $\Omega_{2}$ projection.

Using the tight-binding Hamiltonian[25, 26] with 10 sites, we have implemented the $\chi^{(3)}$ formalism for closing the infinite hierarchy of equations that has been widely applied to Frenkel[27, 28] and Wannier[2, 29] excitons. In Fig. 2, we present the simulated 2D spectra obtained by solving Eqs. 20 and 21 of Ref.[22]) using periodic boundary conditions. Using the same parameters of Ref. [30], we set the dephasing times for excitons $\tau_{e x}=2$ ps and two-excitons $\tau_{2 e x}=1$ ps in panels A-E. Panel E is obtained by cross-circular Gaussian pulses, where $\mathbf{k}_{s}$ is linearly polarized and $\mathbf{k}_{3}, \mathbf{k}_{2}$ and $\mathbf{k}_{1}$ are respectively right, left and right circularly polarized. All other panels are calculated with co-linear Gaussion pulses. Pulse parameters are chosen such that red shifted two-excitons are selectively excited. The optical power spectra width, $\Delta_{o p t}$, and carrier frequency, $\omega_{c}$ (relative to $e$ ) are given in the caption.

In all panels, the origin is $e$ for $\Omega_{3}$ and $2 e$ for $\Omega_{2}$. All peaks or shoulders are assigned using the symbols given in Fig. 1. Circle and solid square are strong and well resolved along $\Omega_{2}$, while solid square and triangle are not resolved along $\Omega_{3}$. The $\Omega_{2}$ value of solid square gives the TBE, $\Delta_{b}$. Panels A and B are calculated respectively with and without correlated two-excitons. As expected, features related to red shifted two-excitons such as the solid square and triangle, disappear. Panels $\mathrm{C}$ and D repeat the calculations of $\mathrm{A}$ and $\mathrm{B}$ for pulses tuned to the blue-shifted two-excitons. One can obtain blue-shifted TSE, $\Delta_{s}$, from the open triangle (a shoulder but not a resolved peak) in panel $\mathrm{C}$, even though we can not 
resolve open square from two-exciton continuum. In panel E (cross-circular excitation), the $\mathrm{HF}$ contribution (the circle) at the origin $(e, 2 e)=(0,0)$ is missing. However, one can still obtain the TBE using the solid square. We also note that TBE may be extracted despite the large line broadening. Panel F shows the 2D spectrum calculated with faster dephasing times $\tau_{e x}=1.0 \mathrm{ps}$ and $\tau_{2 e x}=0.5 \mathrm{ps}$. Even though we can no longer resolve circle and solid square along the clean $\Omega_{2}$ axis, it is still possible to obtain their energies from the 'ridge' of the contour lines (two white lines). The TBE is given by the $\Omega_{2}$ value of the bottom line.

The TBE can be obtained from the intense and well-resolved solid square in panel A. We thus circumvent the difficulty of probing TBE from the splitting between solid square and the unresolved solid triangle, which overlap with $e g$ and $f e$ resonances along $t_{3}$. Additional calculations also show that the TBE related solid square always has a solid triangle to the red, and the TSE related open square always has a open triangle to the blue, as predicted in Fig. 1. Moreover, the solid square and triangle (open square and triangle) always appear in pairs and thus we can easily obtain accurate TBE(TSE) by identifying both features, even when line broadening is large (e.g. panel F). The much weaker solid triangle from pathway (ii) plays a crucial role in identifying the solid square in panel F. Similarly, in panel $\mathrm{C}$ we can not identify the open square without the help of the open triangle. Thus it is impossible to resolve the signature of blue-shifted two-excitons (unresolved open square and triangle) in panel $\mathrm{C}$ with conventional 1D FWM where the signature will either be covered by the two-exciton continuum along $\Omega_{2}$ or along $\Omega_{3}$ by the broadening of the much stronger single-exciton peak, the circle. This signature can not be obtained from any other 2DCS techniques where two-excitons show up along a single axis. However, with the panoramic $2 \mathrm{D}$ view offered by $\mathbf{S}_{\mathbf{I I I}}$, we can easily obtain very accurate TSE, even though the open square and triangle are weak may not be resolved along any single axis.

In $\mathbf{S}_{\mathbf{I}}$, we had demonstrated the first partial separation of two-excitons[22] along $\Omega_{3}$ due to the overlapping $e g$ and $f e$ resonances along $t_{3}$, as shown by the elongation of peaks in both experiments and simulations.[22, 23] $\mathbf{S}_{\mathbf{I I I}}$ provides an additional separation of two-excitons by spreading them along $\Omega_{2}$. Without this separation, one can not resolve any two-exciton features in panel $\mathrm{F}$, let alone the accurate TBE. It is the combination of the two dimensions $\Omega_{3}$ and $\Omega_{2}$ that makes it possible to go from two ambiguous, unresolved open square and triangle in panel $\mathrm{C}$, to retrieve the unique signature of blue-shifted two-excitons and obtain the TSE. 
The $\mathbf{S}_{\mathbf{I I I}}$ technique provides a new perspective into the capacity of TIFWM experiments to provide TBE.[10, 11] Let us recast the existing TIFWM signal using 2DCS terminology. The two-pulse signals[10] along $2 \mathbf{k}_{b}-\mathbf{k}_{a}$ is given by $W_{A}\left(t_{1}\right)=\int_{-\infty}^{+\infty}\left|S_{\mathbf{I}}\left(t_{3}, t_{2}=0, t_{1}\right)\right|^{2} d t_{3}$ (positive delay) and $W_{B}\left(t_{2}\right)=\int_{-\infty}^{+\infty}\left|S_{\mathbf{I I I}}\left(t_{3}, t_{2}, t_{1}=0\right)\right|^{2} d t_{3}$ (negative delay). Three-pulse TIFWM signals[11] along $\mathbf{k}_{a}+\mathbf{k}_{b}-\mathbf{k}_{c}$ correspond to $W_{C}\left(t_{2}\right)=\int_{-\infty}^{+\infty}\left|S_{\text {III }}\left(t_{3}, t_{2}, t_{1}=t_{1}^{0}\right)\right|^{2} d t_{3}$ (positive delay) and $W_{D}\left(t_{2}\right)=\int_{-\infty}^{+\infty}\left|S_{\mathbf{I I}}\left(t_{3}, t_{2}, t_{1}=t_{1}^{0}\right)\right|^{2} d t_{3}$ (negative delay). Strong quantum beats only show up in $W_{B}$ and $W_{C}$, both related to $S_{\text {III }}$ and depend on $t_{2}$. This is clear from our pathway analysis. First, along $t_{2}$ there are only two-exciton resonances $(f g)$ as shown in diagrams (i) and (ii) of Fig. 1, and thus we have well-defined quantum beats along $t_{2}$ and well-resolved peaks along $\Omega_{2}$. Second, the squares from (i) show up mainly as $e g$ resonances along $t_{3}$ and can be much stronger than the triangles, although they are all induced by correlated two-excitons. Without such correlations, there will only be a circle at the origin. It is the stronger solid square and circle that give quantum beats. However, there are no appreciable beats for $W_{A}$ and $W_{D}$. Fig. 3 shows the pathways for $S_{\mathbf{I}}$ and the schematic $2 \mathrm{D}$ spectra projected respectively onto $\left(\Omega_{3},-\Omega_{1}\right)$ and $\left(\Omega_{3}, \Omega_{2}\right)$. Obviously, there are no two-exciton splitting along either $\Omega_{1}$ or $\Omega_{2}$ and thus $S_{\mathbf{I}}$ does not show quantum beats with respect to $t_{1}$ or $t_{2}$, as in $W_{A}$. A similar conclusion applies to $S_{\mathbf{I I}}$, which corresponds to $W_{D}$. The only axis along which quantum beats occur for $S_{\mathbf{I}}$ and $S_{\mathbf{I I}}$ is $t_{3}$. However, it is hard to observe such beats with any technique (including $S_{\mathbf{I I I}}$ ), because there are always dominant $e g$ resonances along $t_{3}$ for all three 2DCS techniques and thus the two-exciton resonances are not well resolved.

$\mathbf{S}_{\text {III }}$ also demonstrates an important limitation of TIFWM in probing TBE. Our calculations show that TIFWM only yields the correct TBE in ideal cases such as Fig. 2A where the $\Omega_{2}$ coordinate of the circle coincides with $2 e$ and its intensity is comparable to solid square. However, the $\Omega_{2}$ value and intensity of the circle are very sensitive to the pulse width, detunings, pulse polarizations, and are further affected by the cancellation of twoexciton continuum to the HF portion. Calculations with long dephasing times (not shown) also suggest that the two-exciton continuum is far from uniform. Although the solid square has a fixed $\Omega_{2}$, its intensity is also very sensitive to the pulse properties. Therefore the circle and solid square do not always dominate the signal. Other components from two-exciton continuum or even open square may also contribute or even dominate the quantum beats. This makes the beating experiment less accurate for probing TBE. Furthermore, in the 
most favorable case for probing TBE where only bound two-excitons are generated (e.g. the cross-circular excitations in Fig. 2E), the HF peak (circle) disappears completely. In this case, any beating frequency obtained is not connected to TBE. Therefore, one has to use other pulse polarizations to obtain quantum beats where the position and intensity of the circle is affected by other types of two-excitons.

The advantage of $\mathbf{S}_{\mathbf{I I I}}$ is even more pronounced when $\mathrm{HH}$ and $\mathrm{LH}$ excitons both contribute and TIFWM signal is congested with many types of beating components along $t_{2}$ arising from pure $\mathrm{HH}(\mathrm{LH})$ and mixed two-excitons. By dispersing all pathways into an extra dimension and spreading them along $\Omega_{2}$ over a spectral range twice that of $\mathbf{S}_{\mathbf{I}}$ and $\mathbf{S}_{\mathbf{I I}}$ techniques, $\mathbf{S}_{\mathbf{I I I}}$ achieves a complete separation of pure $\mathrm{HH}(\mathrm{LH})$ two-excitons from the mixed two-excitons. Furthermore, by choosing specific pulse polarizations, the contribution from correlated pure or mixed two-excitons can be completely isolated from their corresponding HF contributions.

In summary, we propose a 2DCS technique, $S_{\text {III }}$, that is intrinsically sensitive to twoexciton correlations and can distinguish between different species of excitons and twoexcitons in photo-excited semiconductors by spreading them along two axes. Combining the information from different Liouville space pathways,[19] $S_{\text {III }}$ can accurately retrieve TBE even when it is smaller than exciton line broadening. We further report an unambiguous signature of correlated unbound two-excitons lying underneath the two-exciton continuum. Conventional 1D or 2D techniques can not achieve such resolution for the lack of time ordering among pulses, which is essential for the identification of specific pathways. For example, either 1D or 2D pump-probe signals generally mix the $S_{\mathbf{I}}$ and $S_{\mathbf{I I}}$ techniques and thus one can not employ them to identify different pathways, let alone combine them to give higher resolution. $S_{\text {III }}$ also provides new insights into the quantum beats in the most accurate TIFWM for obtaining TBE[10, 11] of quantum wells, which are dominated by one of the two pathways and generally do not provide accurate TBE.

$S_{\text {III }}$ could be applied to other strongly correlated systems such as Bose-Einstein condensate and superconductors. For instance, it could be helpful in the considerable current effort aimed at the coherent manipulation of excitons and exciton Bose-Einstein condensation. Two-exciton effects are critical for achieving these goals.

The support of the Chemical Sciences, Geosciences, and Biosciences Division, Office of Basic Energy Sciences, U. S. Department of Energy is gratefully acknowledged. 
[1] H. Haug and S. W. Koch, Quantum Theory of the Optical and Electronic Properties of Semiconductors, fourth Edition, (World Scientific, 2004).

[2] V. M. Axt and S. Mukamel, Rev. Mod. Phys. 70, 145 (1998).

[3] F. Rossi and T. Kuhn, Rev. of Mod. Phys. 74, 895 (2002).

[4] Th. Östreich, K. Schönhammer, and L. J. Sham, Phys. Rev. B 58, 12920 (1998).

[5] W. Schäfer, D. S. Kim, J. Shah, T. C. Damen, J. E. Cunningham, K. W. Goossen, L. N. Pfeiffer, and K.Köhler, Phys. Rev. B 53, 16429 (1996).

[6] J. Shah, Ultrafast Spectroscopy of Semiconductors and Semiconductor Nanostructures, second enlarged edition, (Springer, 1999).

[7] H. Kalt and M. Hetterich (Eds.), Optics of Semiconductors and their Nanostructures, (Springer, 2004).

[8] V. M. Axt and T. Kuhn, Rep. Prog. Phys. 67, 433-512 (2004).

[9] D. S. Chemla and J. Shah, Nature 411, 549 (2001).

[10] H. Wang, J. Shah, T. C. Damen, L. N. Pfeiffer: Solid State Commun. 91, 869 (1994).

[11] E. J. Mayer, G. O. Smith, V. Heuckeroth, J. Kuhl, K. Bott, A. Schulze, T. Meier, D. Bennhardt, S. W. Koch, P. Thomas, R. Hey, and K. Ploog, Phys. Rev. B 50, 14730 (1994).

[12] M. Koch, J. Feldmann, G. von Plessen, E. O. Göbel, P. Thomas and K. Köhler, Phys. Rev. Lett. 69, 3631 (1992).

[13] S. T. Cundiff, M. Koch, W. H. Knox, J. Shah and W. Stolz, Phys. Rev. Lett. 77, 1107 (1996).

[14] A. Euteneuer, E. Finger, M. Hofmann, W. Stolz, T. Meier, P. Thomas, S. W. Koch, W. W. Rühle, R. Hey and K. Ploog, Phys. Rev. Letts. 83, 2073 (1999).

[15] Ernst R. R., Bodenhausen G., Wokaun A. 1987. Principles of Nuclear Magnetic Resonance in One and Two Dimensions. Oxford, UK: Clarendon.

[16] S. Mukamel, Ann. Rev. Phys. Chem. 51, 691-729 (2000).

[17] M. C. Asplund, M. T. Zanni, R. M. Hochstrasser, Proc. Natl. Acad. Sci. USA 97, 8219 (2000).

[18] T. Brixner, J. Stenger, H. M. Vaswani, M. Cho, R. E. Blankenship and G. R. Fleming, Nature 434, 625-628 (2005).

[19] S. Mukamel, Principles of Nonlinear Optical Spectroscopy, (Oxford University Press, New York, (1995)). 
[20] S. Mukamel and D. Abramavicius, Chem. Rev., 104, 2073-2098 (2004).

[21] Xiaoqin Li, Tianhao Zhang, Camelia N. Borca, and Steven T. Cundiff, Phys. Rev. Lett. 96, 57406 (2006).

[22] L. Yang, I. V. Schweigert, S. T. Cundiff and S. Mukamel, Phys. Rev. B 75, 125302 (2007).

[23] I. Kuznetsova, P. Thomas, T. Meier, T. Zhang, X. Li, R.P. Mirin and S.T. Cundiff, Solid State Communications, Vol 142 (3), 154 (2007).

[24] W. Langbein and B. Patton, J. Phys.: Condens. Matter 19, 295203 (2007).

[25] C. Sieh, T. Meier, F. Jahnke, A. Knorr, S. W. Koch, P. Brick, M. Hübner, C. Ell, J. Prineas, G. Khitrova, and H. M. Gibbs, Phys. Rev. Lett. 82, 3112 (1999).

[26] T. Meier, P. Thomas, and S. W. Koch, Coherent Semiconductor Optics : From Basic Concepts to Nanostructure Applications , (Springer, 2006).

[27] F. C. Spano and S. Mukamel, Phys. Rev. Letts. 66, 1197 (1991).

[28] J. A. Leegwater and S. Mukamel, Phys. Rev. A 46, 452 (1992).

[29] V. M. Axt, and A. Stahl, Z. Phys. B 93, 195 (1994).

[30] S. Weiser, T. Meier, J. Möbius, A. Euteneuer, E. J. Mayer, W. Stolz, M. Hofmann, W. W. Rühle, P. Thomas, and S. W. Koch, Phys. Rev. B 61, 13088 (2000).

Figure captions

Fig. 1 Top: Schematic experimental setup (left) and exciton level scheme (right). Bottom: Feynman diagrams of $S_{\text {III }}$ technique (left) and schematic 2D spectrum (right).

Fig. 2 (Color online) Calculated $\mathbf{S}_{\mathbf{I I I}}\left(\Omega_{3}, \Omega_{2}, t_{1}\right)$ for $(\mathrm{A})$ and $(\mathrm{B}), \Delta_{o p t}=3.9 \mathrm{meV}$, $\omega_{c}=-2.85 \mathrm{meV} ;(\mathrm{C})$ and $(\mathrm{D}), \Delta_{o p t}=1.8 \mathrm{meV}, \omega_{c}=2.15 \mathrm{meV} ;(\mathrm{E}), \Delta_{o p t}=3.9 \mathrm{meV}$, $\omega_{c}=-3.85 \mathrm{meV} ;$ and $(\mathrm{F}) \Delta_{o p t}=3.9 \mathrm{meV}, \omega_{c}=-1.85 \mathrm{meV}$.

Fig. 3 (Color online) Top: Feynman diagrams of $S_{\mathbf{I}}$ technique. Bottom: Schematic 2D spectra, $S_{\mathbf{I}}\left(\Omega_{3}, t_{2},-\Omega_{1}\right)$ (left), $S_{\mathbf{I}}\left(\Omega_{3}, \Omega_{2}, t_{1}\right)$ (right). 
This figure "Fig1.jpeg" is available in "jpeg" format from: http://arxiv.org/ps/0712.2263v1 
This figure "Fig2.jpeg" is available in "jpeg" format from: http://arxiv.org/ps/0712.2263v1 
This figure "Fig3.jpeg" is available in "jpeg" format from: http://arxiv.org/ps/0712.2263v1 